\title{
Socio-Economic Characteristic and Profitability of Sugarcane Cultivation in Ahmednagar District of Maharashtra, India
}

\author{
G.M. Bodakhe $^{1 *}$, S.S. More ${ }^{2}$ and R.D. Shelke ${ }^{1}$ \\ ${ }^{1}$ Department of Agricultural Economics, College of Agriculture, Latur, India \\ ${ }^{2}$ Department of Agricultural Economics, College of Agriculture, Parbhani, India \\ *Corresponding author
}

\section{A B S T R A C T}

Sugarcane occupies a very prominent position on the agricultural map of India. Historically, India is the original home of Saccharum barberi and New Guinea is the Centre of origin of Saccharum officinarum. Sugarcane is an important agro industry crop in India plays a pivotal role in national economic by contributing 1.9 per cent to gross domestic product, form an important component in cropping pattern of the sugarcane growers, provide an essential commodity in the form of sugar and jiggery and solves the problem of green fodder for half of the year. The crop is cultivated in 5.03 million hectare producing more than 357.67 million tones with a productivity of about 69.20 tonnes per hectare. In India, Maharashtra contributes about 45 per cent in area (9.02 lakh hectare) and production (117.14 million tonnes) with 47.36 per cent of total sugarcane production. On the basis of high area under sugarcane crop Shevgaon and Newasa tehsils were selected. From selected two tehsils 6 villages were selected purposely on the basis of highest area under sugarcane crop. From each village 20 sugarcane growers were selected. In this way 120 sugarcane growers were selected for the present study. The aim of this study is to estimate the socio-economic characteristics and per hectare profitability of sugarcane production on different planting season in Ahmednagar district of Maharashtra states in India. The socio-economic characteristics such as average family size, education status, cast composition, cropping pattern, components of sugarcane farm and the analysis of per hectare profitability of sugarcane crop indicated that cultivation of all type of sugarcane were profitable at all the levels of cost, as indicated by into benefit-cost ratio of Adsali, pre-seasonal, suru and ratoon is 1.8,1.7,1.7,1.8 respectively. In adsali, pre-seasonal, suru and ratoon sugarcane group per hectare main produce was 130, 112, 90, 72 tonne and by produce was $12.70,9.50,8.00,7.20$ tonne respectively.

\section{Introduction}

The India is one of the largest sugarcane producers in the world. The sugarcane is an important cash crop grown in India. The sugarcane cultivation and development of sugar industry runs parallel to the growth of human civilization and is as old as agriculture. Sugarcane occupies a prominent position as a cash and commercial crop. Sugarcane is mainly used for making white sugar, gur, khandsari and it is also used for chewing and making juice. In the early days, sugarcane was used for making gur for the consumption of 
common people. In India sugarcane is the third largest crop in the country, in terms of value next to rice and wheat. In order to tackle the expected global energy crisis, many countries are spending considerable money and time in finding alternate renewable source of energy. In such efforts, Ethanol; derived from sugarcane has been found to be a good substitute. In terms of potential productivity of alcohol, sugarcane stands first, compared to other crops. Brazil has forged ahead in the commercial exploitation of sugarcane. In Brazil, most of the vehicles including heavy duty trucks run on gasohol derived from sugarcane. The importance of sugarcane as an energy crop has been realized in our country. Sugarcane is cultivated in more than 110 countries and India stands first in sugar production with around 450 established sugar factories and over 35 million farmers and agricultural labourers involved in sugarcane cultivation and harvesting. Whereas sugarcane is one of the best commercial crops in many parts of India and Maharashtra. While sugarcane is the second largest agro industrial crop with heavy investment and number of varieties of sugarcane are grown in different areas of the Maharashtra State. The price for the sugarcane supplied to the sugar factory is fixed by the local state governments based on the cost of cultivation and other and the price is linked to the sugar recovery.

The Maharashtra State Government has (year 2017-2018) fixed a price of Rs.2500 per tonnes of cane with 11.5 per cent sugar recovery. The sugar factories make payment to the registered cane growers through banks. The sugar industry provides direct employment to a large number of persons, apart from providing indirect employment to thousands of persons in the rural areas who are involved in cultivation, harvesting and transportation of cane and other related services. At present, Maharashtra has practices.
To study the Socio-economic characteristics and profitability of sugarcane cultivation.

\section{Materials and Methods}

The nature of data used for study primary data. Multistage sampling technique is use in the sample selection process. The levels are selection of district, selection of sample villages and selection of sample farmers. The State of Maharashtra is one of the major sugarcane growing States in India. In this State there are farmers whose sells the harvested sugarcane to the sugar factories and at the same time there are farmers who process the sugarcane manufacture Gur. In Maharashtra State, Ahmednagar district is one of the major sugarcane growing district in Maharashtra. Further, in Ahmednagar district there are nine sugar factories and one huge famous Gur marketing yard. In view of these two facts the Ahmednagar district is selected for the study.

The list of sugarcane growers from the selected village were obtained from the revenue records maintained at selected villages and then categorized into four groups according to planting types of sugarcane. Five farmers from each type i.e. Adsali, Suru, Preseasonal and Ratoon sugarcane were selected separately from each village from both the tahsils by simple random sampling technique. Thus, 20 farmers were selected from each village. In all 120 sugarcane growers comprising 30 farmers from each planting type of sugarcane were selected separately for the present study. The primary data was collected from sample sugarcane growers by the survey method. The sample sugarcane growers were contacted individually for collection of required information. The schedule was prepared by keeping in view the objectives of the study. The schedule was pretested by contacting few cultivator and required quantification were made in the 
schedule used for data collection. The field level data / information on the use of various inputs viz., seed, manures, fertilizers, number of irrigations, labour use pattern and yields obtained from sugarcane cultivation, constraints in the use of inputs etc., and general information of sample cultivators, such as family composition, land utilization, cropping pattern and assets position of the farmers etc., were collected from the sample sugarcane growers.

The information on research station yields and yields of field level demonstration plot were obtained from the Central Research Station Padegaon (M. S.) The data were collected for the crop harvested in the year 2017-18.The plantation type wise viz., Suru, Pre-seasonal, Adsali and Ratoon use levels of various inputs and outputs obtained in sugarcane cultivation and the yield gap were estimated by simple tabular method with the help of means, averages and percentages, etc., by keeping in view the objectives of study.

\section{Results and Discussion}

\section{Socio- economic characteristics of sugarcane grower}

Socio-economic aspects of the farmers can be looked upon from different points of view depending upon the number of variables related to their level of living, the socioeconomic environment in which they live and the nature and extent of the farmers participation in developmental activities. Socio-economic characters of the farmers affect their production pattern. The decision making behavior is also determined to a large extent by it. The socio-economic characteristics of the sample farmers considered in this study were composition of family size, level of education, cast composition, cropping patterns, component of farm.

\section{Family size and its composition}

Size of family affects the economic behavior of the family, particularly related to the income and expenditure. A large family is likely to be more conscious for their employment and they need more income to maintain their family. The family size and its composition give an idea about available labour force and their consumption need. The information of sample sugarcane farms about their family size and its composition is presented in Table 1.

As per there are no significant differences among the sample sugarcane grower farms of different planting types except ratoon results are discussed mostly on overall considerations. It is seen from Table 1 that, average size of sugarcane growers family was 6.02 members, consisting of 42.29 per cent males, 35.28 per cent females and 22.38 per cent children. The average family size of ratoon cultivations farm was 8.53, which is slightly higher compared to other. The composition of male, female and children were 42.20, 33.99 and 24.61 percent respectively.

\section{Educational status}

Education is the integral part of any development. Education helps the respondents to know about the adoption of improved practices, which directly affects the production. A literate farmer has better know how about the modern technologies better than illiterate farmers. The total population of the respondents was categorized on the basis of their level of education such as viz. illiterate, primary, secondary and higher secondary and above. The information regarding educational status of the family heads of all the four planting types of sugarcane cultivation is presented in Table 2. 
The Table 2 shows that, only 12.49 per cent farmers are illiterate and literate amongst the heads sample sugarcane growers were 87.50 per cent. Among the literates, majority of the farmers i.e.36.66 per cent have completed secondary education. Farmers with primary and higher secondary and above education accounts for 20.86 per cent and 30.00 per cent respectively. The education status of family heads varies within the sugarcane cultivation i.e. adsali, pre-seasonal, suru and ratoon. The maximum literate farmers were observed in adsali and ratoon cultivation compared to preseasonal and suru cultivation.

\section{Caste}

Caste system is unique in Indian society and also forms the basis of inequality and exploitation. The information regarding cast status of farmer from all the four planting types of sugarcane cultivation is presented in Table 3 and this was believed to be the case particularly in rural India where caste differentiation was seen to be required for the working of the agrarian economy.

In the category the general cast is represented by Marath, muslim, Bhramhn, Marwadi. The backward class is represented by Kumbhar, Vanjari, Dhangar, Hatkar and Lamani. The Scheduled caste is represented by Chambhar, Mang, Bhil and Mahar (Kendre, 2011). It is evident from table 3 that, the maximum 61.66 per cent farmers belonged to general caste followed by 24.16 per cent and 14.16 per cent belonging to backward caste and scheduled caste, respectively.

In case of Adsali sugarcane growers, maximum i.e.76.66 per cent farmers belonged to general caste and 13.33 per cent belonged to backward caste and 10.00 per cent belonged to scheduled caste. In case of Suru sugarcane growers, maximum 50.00 per cent belonged to general caste followed by 26.66 and 23.66 per cent belonging to backward caste and scheduled caste, respectively. Thus, it is clear from table 3 that, the most of the sugarcane growers belonged to general caste. It means general caste people were growing sugarcane as a cash crop, which will be helpful for upliftment of the socio-economic status of sugarcane growers. It is observed from the results that, in all planting of sugarcane cultivation, maximum farmer belongs from general category. This is show that, still the general category holds the land holding and doing the farming business primary.

\section{Cropping pattern}

Cropping pattern of the region is the most important factor in deciding the economic status of the region. Therefore, it assumes the special importance in taking consideration of the crop that would be grown within the environment of the study region. Study of cropping pattern reveals an allocation of land under different crops. Mostly farmers plan their cropping pattern by considering their needs for family requirement and livestock etc. The details of planting type wise area allocation for different crops on the sample farms are presented in Table 4.

At the overall level the average per farm total cropped area was 3.94 hectares. It was observed that the sugarcane occupied a dominant position in the cropping pattern contributing about 40.00 per cent to the total cropped area. Next to sugarcane, cotton occupied second position with share of 11.92 per cent in the total cropped area. Next to sugarcane and cotton, cereals occupied third position with 8.88 per cent. The cereals wheat, bajra and maize were the important crops. Over all Pulses, oilseeds occupied 3.04 per cent and 2.79 Per cent of total cropped area, respectively while, fruits and vegetables crops occupied about $27.66 \%$ per cent area in the cropping pattern. 
At the overall level, gram was the major pulses crop grown in the study area, while groundnut was the major oilseed crops taken in study area. The total area under fruits and vegetables crops were (27.66) per cent and forage crope where (1.01 per cent) of the gross cropped area, respectively at the overall level. More or less similar type of cropping pattern was observed on the sugarcane farms of different planting types viz., Adsali, Pre- seasonal, Suru and Ratoon sugarcane. The cropping pattern of sample sugarcane farms indicated that, farmers have allocated relatively more area under sugarcane crop as compared to other competitions crops. It was because, more sugar factories are established, in the area, availability of canal and lift irrigation facilities in the area under study and better prices for sugarcane in the reasons.

Table.1 Average family size and its composition on sample sugarcane farms

\begin{tabular}{|c|c|c|c|c|c|c|}
\hline \multirow[t]{2}{*}{ Sr. No. } & \multirow[t]{2}{*}{ Particulars } & \multicolumn{4}{|c|}{ Planting type } & \multirow[t]{2}{*}{ Overall } \\
\hline & & Adsali & Pre-seasonal & Suru & Ratoon & \\
\hline 1 & Male & $\begin{array}{c}2.21 \\
(42.41)\end{array}$ & $\begin{array}{c}2.26 \\
(41.69)\end{array}$ & $\begin{array}{c}2.10 \\
(42.87)\end{array}$ & $\begin{array}{c}3.60 \\
(42.20)\end{array}$ & $\begin{array}{c}2.54 \\
(42.29)\end{array}$ \\
\hline 2 & Female & $\begin{array}{c}1.74 \\
(33.39)\end{array}$ & $\begin{array}{c}2.03 \\
(37.45)\end{array}$ & $\begin{array}{c}1.80 \\
(36.79)\end{array}$ & $\begin{array}{c}2.90 \\
(33.99)\end{array}$ & $\begin{array}{c}2.11 \\
(35.28)\end{array}$ \\
\hline 3 & Children & $\begin{array}{c}1.26 \\
(23.28)\end{array}$ & $\begin{array}{c}1.13 \\
(20.84)\end{array}$ & $\begin{array}{c}1.00 \\
(20.64)\end{array}$ & $\begin{array}{c}2.10 \\
(24.61)\end{array}$ & $\begin{array}{c}1.37 \\
(22.38)\end{array}$ \\
\hline & Total & $\begin{array}{c}5.21 \\
(100.00)\end{array}$ & $\begin{array}{c}5.42 \\
(100.00)\end{array}$ & $\begin{array}{c}4.90 \\
(100.00)\end{array}$ & $\begin{array}{c}8.53 \\
(100.00)\end{array}$ & $\begin{array}{c}6.02 \\
(100.00)\end{array}$ \\
\hline
\end{tabular}

(Figures in parentheses indicate the percentages to total)

Table.2 Educational status of family heads of sugarcane cultivators

\begin{tabular}{|c|c|c|c|c|c|c|}
\hline \multirow{2}{*}{$\begin{array}{l}\text { Sr. } \\
\text { No. }\end{array}$} & \multirow[t]{2}{*}{ Particulars } & \multicolumn{4}{|c|}{ Planting type } & \multirow[t]{2}{*}{ Overall } \\
\hline & & Adsali & Preseasonal & Suru & Ratoon & \\
\hline $\mathbf{1}$ & Illiterate & $\begin{array}{l}3.00 \\
(10.00)\end{array}$ & $\begin{array}{l}5.00 \\
(16.66)\end{array}$ & $\begin{array}{l}4.00 \\
(13.33)\end{array}$ & $\begin{array}{l}3.00 \\
(10.00)\end{array}$ & $\begin{array}{l}3.75 \\
(12.49)\end{array}$ \\
\hline 2 & Literate & $\begin{array}{l}27.00 \\
(90.00)\end{array}$ & $\begin{array}{l}25.00 \\
(83.33)\end{array}$ & $\begin{array}{l}26.00 \\
(86.67)\end{array}$ & $\begin{array}{l}27.00 \\
(90.00)\end{array}$ & $\begin{array}{l}26.25 \\
(87.50)\end{array}$ \\
\hline 3 & Up to Primary & $\begin{array}{l}6.00 \\
(20.00)\end{array}$ & $\begin{array}{l}5.00 \\
(16.67)\end{array}$ & $\begin{array}{l}8.00 \\
(26.66)\end{array}$ & $\begin{array}{l}6.00 \\
(20.00)\end{array}$ & $\begin{array}{l}6.25 \\
(20.86)\end{array}$ \\
\hline 4 & Up to Secondary & $\begin{array}{l}11.00 \\
(36.66)\end{array}$ & $\begin{array}{l}12.00 \\
(40.00)\end{array}$ & $\begin{array}{l}10.00 \\
(33.33)\end{array}$ & $\begin{array}{l}11.00 \\
(36.66)\end{array}$ & $\begin{array}{l}11.00 \\
(36.66)\end{array}$ \\
\hline \multirow[t]{2}{*}{5} & Upto Graduate & $\begin{array}{l}10.00 \\
(33.34)\end{array}$ & $\begin{array}{l}8.00 \\
(26.66)\end{array}$ & $\begin{array}{l}8.00 \\
(26.67)\end{array}$ & $\begin{array}{l}10.00 \\
(33.33)\end{array}$ & $\begin{array}{l}9.00 \\
(30.00)\end{array}$ \\
\hline & Total & $\begin{array}{l}30.00 \\
(100.00)\end{array}$ & $\begin{array}{l}30.00 \\
(100.00)\end{array}$ & $\begin{array}{l}30.00 \\
(100.00)\end{array}$ & $\begin{array}{l}30.00 \\
(100.00)\end{array}$ & $\begin{array}{l}120.00 \\
(100.00)\end{array}$ \\
\hline
\end{tabular}

(Figures in parentheses indicate the percentages to total) 
Int.J.Curr.Microbiol.App.Sci (2019) 8(7): 552-564

Table.3 Caste composition of the sugarcane growers

\begin{tabular}{|c|c|c|c|c|c|c|}
\hline \multirow{2}{*}{$\begin{array}{l}\text { Sr. } \\
\text { No. }\end{array}$} & \multirow[t]{2}{*}{ Particulars } & \multicolumn{4}{|c|}{ Planting type } & \multirow[t]{2}{*}{ Overall } \\
\hline & & Adsali & Pre-seasonal & Suru & Ratoon & \\
\hline 1 & General caste & $\begin{array}{c}23.00 \\
(76.66)\end{array}$ & $\begin{array}{c}20.00 \\
(66.66)\end{array}$ & $\begin{array}{c}15.00 \\
(50.00)\end{array}$ & $\begin{array}{c}16.00 \\
(53.33)\end{array}$ & $\begin{array}{c}18.50 \\
(61.66)\end{array}$ \\
\hline 2 & Backward caste & $\begin{array}{c}4.00 \\
(13.33)\end{array}$ & $\begin{array}{c}8.00 \\
(26.56)\end{array}$ & $\begin{array}{c}8.00 \\
(26.66)\end{array}$ & $\begin{array}{c}9.00 \\
(30.00)\end{array}$ & $\begin{array}{c}7.25 \\
(24.16)\end{array}$ \\
\hline 3 & Scheduled caste & $\begin{array}{c}3.00 \\
(10.00)\end{array}$ & $\begin{array}{c}2.00 \\
(6.76)\end{array}$ & $\begin{array}{c}7.00 \\
(23.33)\end{array}$ & $\begin{array}{c}5.00 \\
(16.66)\end{array}$ & $\begin{array}{c}4.25 \\
(14.16)\end{array}$ \\
\hline & Total & $\begin{array}{l}30.00 \\
(100.00)\end{array}$ & $\begin{array}{l}30.00 \\
(100.00)\end{array}$ & $\begin{array}{l}30.00 \\
(100.00)\end{array}$ & $\begin{array}{l}30.00 \\
(100.00)\end{array}$ & $\begin{array}{l}30.00 \\
(100.00)\end{array}$ \\
\hline
\end{tabular}

(Figures in parentheses indicate the percentages to total respective holding)

Table.4 Cropping pattern of sugarcane cultivators

(ha)

\begin{tabular}{|c|c|c|c|c|c|c|}
\hline \multirow[t]{2}{*}{ Sr. No. } & \multirow[t]{2}{*}{ Particulars } & \multicolumn{4}{|c|}{ Planting types } & \multirow[t]{2}{*}{ Overall } \\
\hline & & Adsali & Pre-seasonal & Suru & Ratoon & \\
\hline 1 & Cereals & $\begin{array}{c}0.46 \\
(9.58)\end{array}$ & $\begin{array}{c}0.37 \\
(9.20)\end{array}$ & $\begin{array}{c}0.22 \\
(6.48)\end{array}$ & $\begin{array}{c}0.37 \\
(10.69)\end{array}$ & $\begin{array}{c}0.35 \\
(8.88)\end{array}$ \\
\hline 2 & Pulses & $\begin{array}{l}0.15 \\
(3.12)\end{array}$ & $\begin{array}{c}0.11 \\
(9.44)\end{array}$ & $\begin{array}{c}0.10 \\
(2.94)\end{array}$ & $\begin{array}{c}0.12 \\
(3.46)\end{array}$ & $\begin{array}{c}0.12 \\
(3.04)\end{array}$ \\
\hline 3 & $\begin{array}{l}\text { Total Food } \\
\text { Grains }\end{array}$ & $\begin{array}{c}0.61 \\
(12.70)\end{array}$ & $\begin{array}{c}0.48 \\
(11.94)\end{array}$ & $\begin{array}{c}0.32 \\
(9.43)\end{array}$ & $\begin{array}{c}0.49 \\
(14.16)\end{array}$ & $\begin{array}{c}0.47 \\
(\mathbf{1 1 . 9 2})\end{array}$ \\
\hline 4 & Oil Seeds & $\begin{array}{c}0.18 \\
(3.75)\end{array}$ & $\begin{array}{c}0.10 \\
(2.48)\end{array}$ & $\begin{array}{c}0.12 \\
(3.53)\end{array}$ & $\begin{array}{c}0.07 \\
(2.02)\end{array}$ & $\begin{array}{c}0.11 \\
(2.79)\end{array}$ \\
\hline 5 & $\begin{array}{l}\text { Fruits and } \\
\text { Vegetables }\end{array}$ & $\begin{array}{c}1.33 \\
(27.70)\end{array}$ & $\begin{array}{c}1.15 \\
(28.60)\end{array}$ & $\begin{array}{c}0.99 \\
(20.35)\end{array}$ & $\begin{array}{c}1.17 \\
(33.81)\end{array}$ & $\begin{array}{c}1.09 \\
(27.66)\end{array}$ \\
\hline 6 & Cotton & $\begin{array}{c}0.63 \\
(13.12)\end{array}$ & $\begin{array}{c}0.59 \\
(14.67)\end{array}$ & $\begin{array}{c}0.45 \\
(10.32)\end{array}$ & $\begin{array}{c}0.32 \\
(9.24)\end{array}$ & $\begin{array}{c}0.47 \\
(11.92)\end{array}$ \\
\hline 7 & Forage crops & $\begin{array}{c}0.01 \\
(0.20)\end{array}$ & $\begin{array}{c}0.05 \\
(1.27)\end{array}$ & $\begin{array}{c}0.02 \\
(0.58)\end{array}$ & $\begin{array}{c}0.10 \\
(2.89)\end{array}$ & $\begin{array}{c}0.04 \\
(1.01)\end{array}$ \\
\hline 8 & Sugarcane & $\begin{array}{c}2.05 \\
(42.70)\end{array}$ & $\begin{array}{c}1.65 \\
(41.04)\end{array}$ & $\begin{array}{c}1.26 \\
(37.16)\end{array}$ & $\begin{array}{c}1.31 \\
(37.86)\end{array}$ & $\begin{array}{c}1.56 \\
(39.59)\end{array}$ \\
\hline & $\begin{array}{l}\text { Total Cropped } \\
\text { Area }\end{array}$ & $\begin{array}{c}4.80 \\
(100.00)\end{array}$ & $\begin{array}{c}4.03 \\
(100.00)\end{array}$ & $\begin{array}{c}3.39 \\
(100.00)\end{array}$ & $\begin{array}{c}3.46 \\
(100.00)\end{array}$ & $\begin{array}{c}3.94 \\
(100.00)\end{array}$ \\
\hline & $\mathrm{NCA}$ & 3.28 & 3.07 & 2.12 & 2.41 & 2.65 \\
\hline & $\begin{array}{l}\text { Cropping } \\
\text { intensity }(\%)\end{array}$ & 146.40 & 130.43 & 159.90 & 156.70 & 148.67 \\
\hline
\end{tabular}

(Figures in parentheses indicate the percentages to respective total cropped area) 
Int.J.Curr.Microbiol.App.Sci (2019) 8(7): 552-564

Table.5 Components of sugarcane farms

\begin{tabular}{|c|c|c|c|c|c|c|}
\hline \multirow{2}{*}{$\begin{array}{l}\text { Sr. } \\
\text { No }\end{array}$} & \multirow[t]{2}{*}{ Particulars } & \multicolumn{4}{|c|}{ Planting types } & \multirow[t]{2}{*}{ Overall } \\
\hline & & Adsali & Pre- & Suru & Ratoon & \\
\hline & & & seasonal & & & \\
\hline 1 & Land holdings & 3.59 & 3.40 & 2.45 & 2.61 & 3.01 \\
\hline 2 & $\begin{array}{l}\text { Buildings } \\
\text { (sq.feet) }\end{array}$ & 1225 & 1024 & 1250 & 1120 & 1154 \\
\hline 3 & $\begin{array}{l}\text { Cattle shed } \\
\text { (sq.feet) }\end{array}$ & 400 & 360 & 480 & 382 & 405 \\
\hline \multirow[t]{4}{*}{4} & $\begin{array}{l}\text { Irrigation } \\
\text { structure }\end{array}$ & 1.20 & 1.03 & 0.96 & 1.30 & 1.12 \\
\hline & Well & 1.23 & 1.02 & 1.15 & 1.32 & 1.18 \\
\hline & Tube well & 0.86 & 1.16 & 1.03 & 0.96 & 1.00 \\
\hline & Drip irrigation & 0.60 & 0.72 & 0.65 & 0.56 & 0.63 \\
\hline \multirow[t]{6}{*}{5} & $\begin{array}{l}\text { Implements and } \\
\text { machinery }\end{array}$ & 0.63 & 0.72 & 0.55 & 0.68 & 0.64 \\
\hline & Tractor & 0.45 & 0.39 & 0.56 & 0.41 & 0.45 \\
\hline & Sprear & 0.32 & 0.26 & 0.28 & 0.35 & 0.30 \\
\hline & Iron plough & 0.41 & 0.38 & 0.43 & 0.38 & 0.41 \\
\hline & Seed dril & 0.46 & 1.09 & 0.68 & 0.59 & 0.70 \\
\hline & Harrow & 0.41 & 0.35 & 0.42 & 035 & 0.38 \\
\hline \multirow[t]{6}{*}{6} & Livestock & & & & & \\
\hline & Bullock pair & 0.83 & 0.46 & 0.63 & 0.58 & 0.62 \\
\hline & Buffalo & 2.12 & 1.80 & 2.68 & 3.08 & 2.42 \\
\hline & Cow & 4.64 & 5.33 & 5.41 & 3.28 & 4.66 \\
\hline & Goat & 2.70 & 3.42 & 4.18 & 4.15 & 3.41 \\
\hline & Poultry & 2.58 & 4.12 & 3.88 & 3.64 & 3.55 \\
\hline
\end{tabular}


Table.6 Per hectare physical input utilization in sugarcane cultivation:

\begin{tabular}{|c|c|c|c|c|c|c|}
\hline \multirow{2}{*}{$\begin{array}{l}\text { Sr. } \\
\text { No }\end{array}$} & \multirow{2}{*}{$\begin{array}{c}\text { Particulars } \\
\text { (days) }\end{array}$} & \multicolumn{4}{|c|}{ Planting types } & \multirow{2}{*}{$\begin{array}{l}\text { Overall } \\
\text { (Rs.) }\end{array}$} \\
\hline & & Adsali & $\begin{array}{c}\text { Pre- } \\
\text { seasonal }\end{array}$ & Suru & Ratoon & \\
\hline \multirow[t]{4}{*}{1} & Hired labour & & & & & \\
\hline & Male & 92.56 & 79.37 & 68.23 & 56.23 & 74.34 \\
\hline & Female & 71.67 & 63.27 & 54.73 & 52.54 & 60.55 \\
\hline & Total & 164.23 & 142.64 & 122.00 & 108.77 & 134.41 \\
\hline \multirow[t]{4}{*}{2} & Family labour & & & & & \\
\hline & Male & 64.54 & 60.12 & 55.16 & 46.43 & 56.56 \\
\hline & Female & 30.12 & 26.12 & 28.13 & 32.12 & 29.12 \\
\hline & Total & 94.66 & 86.24 & 83.29 & 78.55 & 85.68 \\
\hline \multirow[t]{4}{*}{3} & Total labour & & & & & \\
\hline & Male & 157.10 & 139.49 & 123.39 & 102.66 & 130.16 \\
\hline & Female & 101.79 & 89.39 & 82.86 & 84.66 & 89.67 \\
\hline & Total & 258.89 & 228.88 & 205.25 & 187.32 & 220.08 \\
\hline 4 & $\begin{array}{l}\text { Machine power } \\
\text { (hrs) }\end{array}$ & 27.81 & 30.12 & 26.78 & 16.18 & 24.75 \\
\hline 5 & Bullock labour & 4.70 & 3.65 & 2.64 & 2.87 & 3.44 \\
\hline 6 & $\begin{array}{l}\text { Planting } \\
\text { material (tonne) }\end{array}$ & 4.49 & 3.54 & 2.68 & - & 2.67 \\
\hline 7 & $\begin{array}{l}\text { Manures } \\
\text { (tonne) }\end{array}$ & 18.17 & 11.89 & 7.57 & 7.72 & 11.33 \\
\hline \multirow[t]{4}{*}{8} & Fertilizers (kg) & & & & & \\
\hline & $\mathrm{N}$ & 381.60 & 242.40 & 158.40 & 166.00 & 237.10 \\
\hline & $\mathrm{P}$ & 180.00 & 123.60 & 79.60 & 82.00 & 116.32 \\
\hline & $\mathrm{K}$ & 174.40 & 122.40 & 78.80 & 77.60 & 113.30 \\
\hline 9 & $\begin{array}{l}\text { Plant protection } \\
\text { (lt.) }\end{array}$ & 0.19 & 0.19 & 0.25 & 0.16 & 0.79 \\
\hline 10 & $\begin{array}{l}\text { Micro nutrients } \\
\text { (kg) }\end{array}$ & 12 & 10 & 8 & 9 & 9.75 \\
\hline
\end{tabular}


Table.7 Per hectare physical input utilization in sugarcane cultivation:

\begin{tabular}{|c|c|c|c|c|c|c|}
\hline \multirow{2}{*}{$\begin{array}{l}\text { Sr. } \\
\text { No }\end{array}$} & \multirow[t]{2}{*}{ Particulars } & \multicolumn{4}{|c|}{ Planting types } & \multirow{2}{*}{$\begin{array}{l}\text { Overall } \\
\text { (Rs.) }\end{array}$} \\
\hline & & Adsali & Pre-seasonal & Suru & Ratoon & \\
\hline \multirow[t]{4}{*}{1} & Hired labour & & & & & \\
\hline & Male (man days) & $\begin{array}{l}23140 \\
(11.88)\end{array}$ & $\begin{array}{c}19842 \\
(12.65)\end{array}$ & $\begin{array}{c}17000 \\
(13.45)\end{array}$ & $\begin{array}{l}14057 \\
(9.23)\end{array}$ & $\begin{array}{l}18509.8 \\
(12.45)\end{array}$ \\
\hline & Female & $\begin{array}{l}10750 \\
(4.42)\end{array}$ & $\begin{array}{l}9490 \\
(5.36)\end{array}$ & $\begin{array}{c}8209 \\
(5.46)\end{array}$ & $\begin{array}{c}7881 \\
(7.23)\end{array}$ & $\begin{array}{l}9082 \\
(5.13)\end{array}$ \\
\hline & Total & $\begin{array}{c}33890 \\
(16.27)\end{array}$ & $\begin{array}{c}29332 \\
(18.84)\end{array}$ & $\begin{array}{l}25209 \\
(18.49)\end{array}$ & $\begin{array}{c}21938 \\
(17.90)\end{array}$ & $\begin{array}{c}27592.3 \\
(18.20)\end{array}$ \\
\hline 2 & Machine power (hours) & $\begin{array}{l}10848 \\
(4.52)\end{array}$ & $\begin{array}{l}12048 \\
(5.13)\end{array}$ & $\begin{array}{l}10712 \\
(4 . .30)\end{array}$ & $\begin{array}{c}6472 \\
(3.12) \\
\end{array}$ & $\begin{array}{c}9900 \\
(4.88)\end{array}$ \\
\hline 3 & $\begin{array}{l}\text { Bullock labour } \\
\text { (pairs day) }\end{array}$ & $\begin{array}{l}3780 \\
(2.03) \\
\end{array}$ & $\begin{array}{l}2920 \\
(1.97)\end{array}$ & $\begin{array}{c}2112 \\
(1.80)\end{array}$ & $\begin{array}{l}2296 \\
(2.39)\end{array}$ & $\begin{array}{l}2777 \\
(2.03)\end{array}$ \\
\hline 4 & Planting material (tonne) & $\begin{array}{l}11252 \\
(6.06)\end{array}$ & $\begin{array}{l}9850 \\
(6.66)\end{array}$ & $\begin{array}{c}6700 \\
(5.71)\end{array}$ & - & $\begin{array}{l}6975 \\
(5.08)\end{array}$ \\
\hline 5 & Manures(tonne) & $\begin{array}{c}27255 \\
(14.59)\end{array}$ & $\begin{array}{l}22835 \\
(15.44)\end{array}$ & $\begin{array}{l}15355 \\
(13.09)\end{array}$ & $\begin{array}{c}11580 \\
(12.09)\end{array}$ & $\begin{array}{l}19256.3 \\
(14.10)\end{array}$ \\
\hline \multirow[t]{5}{*}{6} & Fertilizers (Kg) & & & & & \\
\hline & $\mathrm{N}$ & $\begin{array}{l}4960 \\
(2.67)\end{array}$ & $\begin{array}{l}4151 \\
(2.80)\end{array}$ & $\begin{array}{c}2059 \\
(1.75)\end{array}$ & $\begin{array}{l}2158 \\
(2.25)\end{array}$ & $\begin{array}{c}3332 \\
(2.43)\end{array}$ \\
\hline & $\mathrm{P}$ & $\begin{array}{l}7200 \\
(3.88)\end{array}$ & $\begin{array}{c}6920 \\
(4.80)\end{array}$ & $\begin{array}{l}3160 \\
(2.69)\end{array}$ & $\begin{array}{c}3280 \\
(3.66)\end{array}$ & $\begin{array}{l}5140 \\
(3.76)\end{array}$ \\
\hline & $\mathrm{K}$ & $\begin{array}{l}4525 \\
(2.29)\end{array}$ & $\begin{array}{c}3172 \\
(2.68)\end{array}$ & $\begin{array}{c}2028 \\
(1.72)\end{array}$ & $\begin{array}{c}2002 \\
(2.09)\end{array}$ & $\begin{array}{l}2931 \\
(2.14)\end{array}$ \\
\hline & Micro nutrients (kg) & $\begin{array}{c}600 \\
(0.30)\end{array}$ & $\begin{array}{c}550 \\
(0.24)\end{array}$ & $\begin{array}{c}450 \\
(0.20)\end{array}$ & $\begin{array}{c}400 \\
(0.16)\end{array}$ & $\begin{array}{l}487.5 \\
(0.22)\end{array}$ \\
\hline 7 & Plant protection (lt.) & $\begin{array}{r}1150 \\
(0.62)\end{array}$ & $\begin{array}{c}950 \\
(0.64)\end{array}$ & $\begin{array}{c}880 \\
(0.75)\end{array}$ & $\begin{array}{c}860 \\
(0.89)\end{array}$ & $\begin{array}{c}960 \\
(0.71)\end{array}$ \\
\hline \multirow[t]{2}{*}{8} & Irrigation charges (Rs) & $\begin{array}{l}7900 \\
(4.25)\end{array}$ & $\begin{array}{l}6726 \\
(4.55)\end{array}$ & $\begin{array}{c}5000 \\
(4.26)\end{array}$ & $\begin{array}{l}5200 \\
(5.42)\end{array}$ & $\begin{array}{l}6206 \\
(4.54)\end{array}$ \\
\hline & Input cost & $\begin{array}{l}112760 \\
(54.95)\end{array}$ & $\begin{array}{l}98904 \\
(58.75)\end{array}$ & $\begin{array}{l}73912 \\
(53.23)\end{array}$ & $\begin{array}{l}55786 \\
(51.48)\end{array}$ & $\begin{array}{r}85069 \\
(55.02)\end{array}$ \\
\hline 9 & $\begin{array}{l}\text { Land revenue and other cesses } \\
\text { (Rs) }\end{array}$ & $\begin{array}{c}350 \\
(0.18)\end{array}$ & $\begin{array}{c}325 \\
(0.21)\end{array}$ & $\begin{array}{c}300 \\
(0.25)\end{array}$ & $\begin{array}{c}300 \\
(0.31)\end{array}$ & $\begin{array}{c}318 \\
(0.23)\end{array}$ \\
\hline 10 & $\begin{array}{l}\text { Depre. on machinery and } \\
\text { implements (Rs) }\end{array}$ & $\begin{array}{c}705 \\
(0.38)\end{array}$ & $\begin{array}{c}586 \\
(0.39)\end{array}$ & $\begin{array}{c}576 \\
(0.49)\end{array}$ & $\begin{array}{c}470 \\
(0.49)\end{array}$ & $\begin{array}{c}584 \\
(0.42)\end{array}$ \\
\hline 11 & Working capital (Rs) & $\begin{array}{l}114415 \\
(55.52)\end{array}$ & $\begin{array}{l}100365 \\
(59.37)\end{array}$ & $\begin{array}{l}74541 \\
(54.03)\end{array}$ & $\begin{array}{l}56956 \\
(52.29)\end{array}$ & $\begin{array}{l}86459.1 \\
(55.68)\end{array}$ \\
\hline \multirow[t]{2}{*}{12} & Interest on working capital (Rs) & $\begin{array}{l}13385 \\
(7.21)\end{array}$ & $\begin{array}{c}11409.7 \\
(7.71)\end{array}$ & $\begin{array}{l}8239 \\
(7.02)\end{array}$ & $\begin{array}{l}6510 \\
(6.79)\end{array}$ & $\begin{array}{c}9885.93 \\
(7.23)\end{array}$ \\
\hline & Cost A & $\begin{array}{l}127800 \\
(62.73)\end{array}$ & $\begin{array}{l}111775 \\
(66.47)\end{array}$ & $\begin{array}{l}82780 \\
(61.06)\end{array}$ & $\begin{array}{c}63466 \\
(59.58)\end{array}$ & $\begin{array}{c}96345 \\
(62.76)\end{array}$ \\
\hline 13 & $\begin{array}{l}\text { Interest on fixed capital @ } 10 \\
\text { per cent (Rs) }\end{array}$ & $\begin{array}{l}4659 \\
(2.51)\end{array}$ & $\begin{array}{l}4138 \\
(2.79)\end{array}$ & $\begin{array}{c}3182 \\
(2.75)\end{array}$ & $\begin{array}{l}2950 \\
(3.07)\end{array}$ & $\begin{array}{c}3732 \\
(2.73)\end{array}$ \\
\hline \multirow[t]{2}{*}{14} & $\begin{array}{l}\text { Rental value of owened land } \\
\text { (Rs) }\end{array}$ & $\begin{array}{l}43947 \\
(23.69)\end{array}$ & $\begin{array}{c}26517 \\
(17.93)\end{array}$ & $\begin{array}{l}24536 \\
(20.92)\end{array}$ & $\begin{array}{c}19937 \\
(20.81)\end{array}$ & $\begin{array}{l}28734.3 \\
(21.04)\end{array}$ \\
\hline & Cost B & $\begin{array}{l}176406 \\
(88.94)\end{array}$ & $\begin{array}{l}128921 \\
(87.21)\end{array}$ & $\begin{array}{l}110498 \\
(84.70)\end{array}$ & $\begin{array}{c}86353 \\
(82.98)\end{array}$ & $\begin{array}{l}128811 \\
(86.53)\end{array}$ \\
\hline \multirow[t]{5}{*}{15} & Family labour & & & & & \\
\hline & Male (man days) & $\begin{array}{l}16000 \\
(8.62)\end{array}$ & $\begin{array}{l}15000 \\
(10.14)\end{array}$ & $\begin{array}{l}13750 \\
(11.72)\end{array}$ & $\begin{array}{c}11500 \\
(12.84)\end{array}$ & $\begin{array}{l}14062.5 \\
(10.29)\end{array}$ \\
\hline & Female & $\begin{array}{l}4500 \\
(2.42)\end{array}$ & $\begin{array}{l}4900 \\
(3.21)\end{array}$ & $\begin{array}{l}4200 \\
(3.58)\end{array}$ & $\begin{array}{l}4800 \\
(5.01)\end{array}$ & $\begin{array}{l}4600 \\
(3.36)\end{array}$ \\
\hline & Total & $\begin{array}{l}20500 \\
(11.01)\end{array}$ & $\begin{array}{c}18900 \\
(12.75)\end{array}$ & $\begin{array}{c}17950 \\
(15.30)\end{array}$ & $\begin{array}{c}16300 \\
(17.01)\end{array}$ & $\begin{array}{l}18412.5 \\
(13.48)\end{array}$ \\
\hline & Cost C & $\begin{array}{c}196906 \\
(100)\end{array}$ & $\begin{array}{c}161329 \\
(100)\end{array}$ & $\begin{array}{c}128428 \\
(100)\end{array}$ & $\begin{array}{c}102653 \\
(100)\end{array}$ & $\begin{array}{c}147223 \\
(100)\end{array}$ \\
\hline
\end{tabular}


Table.8 Per hectare profitability of sugarcane cultivation:

\begin{tabular}{|c|c|c|c|c|c|c|}
\hline \multirow{2}{*}{$\begin{array}{l}\text { Sr. } \\
\text { No }\end{array}$} & \multirow{2}{*}{$\begin{array}{c}\text { Particulars } \\
\text { (days) }\end{array}$} & \multicolumn{4}{|c|}{ Planting types } & \multirow{2}{*}{$\begin{array}{c}\text { Overall } \\
\text { (Rs.) }\end{array}$} \\
\hline & & Adsali & Pre-seasonal & Suru & Ratoon & \\
\hline \multirow[t]{5}{*}{1} & Production & & & & & \\
\hline & Main produce & & & & & \\
\hline & Quantity (t) & 130 & 112 & 90 & 72 & 101 \\
\hline & Price received (Rs./t) & 2500 & 2500 & 2500 & 2500 & 2500 \\
\hline & Returns(Rs.) & 325000 & 280000 & 225000 & 180000 & 225500 \\
\hline \multirow[t]{5}{*}{2} & By produce & & & & & \\
\hline & Quantity (t) & 12.70 & 9.50 & 8.00 & 7.20 & 10.27 \\
\hline & Price received (Rs./t) & 650 & 650 & 650 & 650 & 650 \\
\hline & Returns(Rs.) & 8255 & 6175 & 5200 & 4680 & 6675 \\
\hline & $\begin{array}{l}\text { Gross returns (i+ii) } \\
\text { (Rs.) }\end{array}$ & 333255 & 286175 & 230200 & 184680 & 232175 \\
\hline \multirow[t]{4}{*}{3} & Costs (Rs.) & & & & & \\
\hline & Cost A (Rs.) & 127800 & 111775 & 82780 & 63466 & 96345 \\
\hline & Cost B (Rs.) & 176406 & 128921 & 110498 & 86353 & 128811 \\
\hline & Cost C (Rs.) & 196906 & 161329 & 128428 & 102653 & 147223 \\
\hline \multirow[t]{4}{*}{4} & Net returns at (Rs.) & & & & & \\
\hline & Cost A (Rs.) & 205455 & 174400 & 147420 & 121214 & 135830 \\
\hline & Cost B (Rs.) & 156849 & 157254 & 119702 & 98327 & 103364 \\
\hline & Cost C (Rs.) & 136349 & 124846 & 101772 & 82027 & 84952 \\
\hline 5 & Cost/tone (Rs.) & 1514.66 & 1440.43 & 1426.97 & 1425.73 & 1457.65 \\
\hline 6 & Cost/quintal (Rs.) & 151.46 & 144.04 & 142.97 & 142.57 & 145.76 \\
\hline 7 & Benefit cost ratio & 1.8 & 1.7 & 1.7 & 1.8 & 1.7 \\
\hline
\end{tabular}

\section{Components of sugarcane Farms}

The information regarding per average total land holting, buildings, Cattle shed, irrigation structure, implements and machinery, livestock use by the sample sugarcane growers is given in Table 5.

Result reported in the table 5 revealed that, the average land holding of sugarcane grower is 3.01 ha. According to planting types, it was highest on Adsali sugarcane grower 3.59 ha followed by pre-seasonal, ratoon and suru i.e. 3.40, 2.61, 2.45 resp. The all planting season sugarcane grower have buildings the average area of building was 1154 sq.feet. In Table 5 it was seen from the result that, all the sugarcane growers had livestock on their farm. The average bullock pair, buffalo, cow, goat and poultry was $0.62,2.42,4.66,3.41$, and 3.55 respectively. The implement and machinery in all planting sugarcane grower was present such as tractor, sprayer, iron plough, harrow and seed dril, was the average is $0.45,0.30,0.41,0.38,0.70$, respectively.

\section{Profitability of sugarcane cultivation}

The profitability of the farm business can be decided from relationship between costs incurred and returns obtained from it. The cost structure depends upon the type of resource employed, the resource mix and the extent of their application. The primary focus 
of this section is therefore, to compare the resource use structure in all the four types of sugarcane planting. The resource use in sugarcane cultivation on per hectare basis in Adsali, Pre-seasonal, Suru and Ratoon sugarcane is presented in Table 6 . The detailed per hectare planting type wise cost of cultivation of sugarcane is presented following table 6 .

\section{Per hectare physical input utilization}

The per hectare physical input utilization for sugarcane cultivation is given in Table 6 .

The per hectare physical input utilization for sugarcane cultivation is given in Table 6. It is observed from the Table 6 that, overall per hectare total human labour used where 220.08 days of which 130.16 male labour and 89.67 female labour. Total human labour used were highest in adsali sugarcane cultivation i.e. 258.89 days, of which 157.10 days were male labour and 101.79 days were female labour and lowest in ratoon sugarcane cultivation which were 187.32 days, among them 102.66 days were male labour and 84.66 days were female labour. Overall per hectare machine power use were $24.75 \mathrm{hrs}$ and highest machinery power use in pre-seasonal sugarcanes cultivation. Overall Per hectare bullock labour used were 3.44 days. Across the planting type i.e. Adsali, pre-seasonal, suru and ratoon the use of bullock labour in sugarcane cultivation was 4.70, 3.65, 2.64, and 2.87 days, respectively.

At overall, per hectare quantity of planting material used was 2.67 tonne, and higest per hectare use planting material was seen in Adsali sugarcane cultivation (4.49 tonne), followed by Pre-seasonal and Suru cultivation was 3.54 and 2.68 tonne. Manures are an important input for sugarcane cultivation, it used to the extent of 11.33 tonne per hectare in overall while adsali, pre-seasonal, suru and ratoon cultivation, it was $18.17,11.89,7.57$, 7.72 tonne per hectare, respectively. The per hectare quantity of fertilizers used in overall sugarcane cultivation was $237.10 \mathrm{~kg}$ of $\mathrm{N}$, $116.32 \mathrm{~kg}$ of $\mathrm{P}$ and $113.30 \mathrm{~kg}$ of $\mathrm{K}$. It was highest in adsali sugarcane cultivation, i.e. $381.60 \mathrm{~kg}$ of N, $180.00 \mathrm{~kg}$ of $\mathrm{P}$ and $174.70 \mathrm{~kg}$ of $\mathrm{K}$, and lowest in ratoon sugarcane cultivation. It was $166.00 \mathrm{~kg}$ of N, $82.00 \mathrm{~kg}$ of $\mathrm{P}$ and $70.66 \mathrm{~kg}$ of $\mathrm{K}$. The use of plant protection was found negligible it was mostly use to control rodents.

\section{Per hectare cost of cultivation of sugarcane}

The item wise and group wise per hectare cost of cultivation of sugarcane is presented in Table 7. It is observed from the Table 7 that, Overall total cost of cultivation of sugarcane was workout to be Rs. 1,47,223, while highest total cost of cultivation (cost C) of Adsali sugarcane was worked out to Rs. 1,96,906, and lowest in ratoon sugarcane it was worked out to Rs. 1,02653, in pre-seasonal and suru sugarcane cultivation total cost of cultivation was worked out to Rs. 1,61,329 and Rs. $1,28,428$, respectively. In Adsali sugarcane of the total cost (cost C), share of cost A was 62.73 per cent and cost B was 88.94 per cent, while in ratoon sugarcane, total cost of cultivation (cost C) was worked out to Rs. $1,02,653$ of which share of cost A was 59.58 per cent and cost B was 82.98 per cent. It was observed from the result that cost $\mathrm{A}$ was minimum (Rs. 63,466) in ratoon sugarcane and it was maximum (Rs. 1,27,800) in Adsaali sugarcane.

In Adsali sugarcane, out of total cost, the most expensive item was rental value of land (23.69\%) followed by hired labour (16.27\%), manures $(14.59 \%)$ fertilizers $(9.11 \%)$, interest on working capital $(7.21 \%)$, planting material (6.06\%) bullock labour (2.03\%), irrigation charges $(4.25 \%)$, interest on fixed capital $(2.51 \%)$, plant protection $(0.62 \%)$, 
depreciation on implements and machinery $(0.38 \%)$ and land revenue and other cesses $(0.18 \%)$.

In ratoon sugarcane crop out of total cost the most expensive item was hired human labour $(18.20 \%)$ followed by rental value of land $(20.81 \%)$, family labour $(17.01 \%)$, manures $(12.09 \%)$, fertilizers $(8.56 \%)$, interest on working capital $(6.79 \%)$, irrigation charges $(5.42 \%), \%)$, interest on fixed capital (3.07\%), bullock labour (2.39\%), plant protection $(0.89 \%)$ land revenue and other cesses $(0.31 \%)$ and depreciation on implements and machinery $(0.49 \%)$. The results reveal that, Cost C was the highest Rs.1,27,800. At overall total hired labour cost is Rs. 27892, machine charges is Rs. 9900, bullock pair charges is Rs.2777, manure chagres is Rs. 19256, fertilizer charge is Rs.11352, plant protection charges is Rs. 960, irrigation charges is Rs.6206. This table is show that the most of the cost are use for hired labour, followed by manures, fertilizer, machine power, irrigation.

\section{Per hectare profitability of sugarcane cultivation}

Per hectare cost, returns and profitability of sugarcane cultivation was worked out as per standard cost concepts and is presented in Table 8. It is seen from the Table 8 that, sugarcane cultivation was found to be profitable in Adsali, pre-seasonal, Suru and ratoon groups. It is observed from the table that, the per hectare sugarcane production in Adsali was 130 tons and in pre-seasonal, suru and ratoon it was 112 tons, 90 tons and 72 tons respectively. Benefit: cost ratio was 1.8 , 1.7, 1.7, 1.8 in Adsali, pre-seasonal suru and ratoon sugarcane, respectively.

It is revealed from the Table 8 that, the price realized by producer was Rs. 2500/tonne for main produce and Rs.650/ tonne for by produce, for Adsali, Pre-seasonal, suru and ratoon sugarcane crop, respectively. In overall sugarcane cultivation, per hectare total cost was Rs. 147223 while in adsali, pre-seasonal, suru and ratoon sugarcane per hectare total cost was Rs. 196906, Rs. 1161329, Rs. 128428 , Rs. 102653 respectively. At overall sugarcane profitability was Rs. 135830 at cost A, Rs. 103364, at cost B and Rs. 84952 at cost C. The net profit (at cost C) was Rs. 84992 in overall sugarcane and was Rs. 136349 in adsali sugarcane which was highest among all planting type. Per tonne cost of production of adsali sugarcane was Rs. 1514.66 while it was Rs. 1440.43 , Rs. 1426.97, Rs. 1425.57 in Pre-seasonal, suru and ratoon sugarcane cultivation. Per quintal cost of production of overall sugarcane was Rs. 145.76 while it was highest in ratoon sugarcane cultivation i.e. Rs. 151.46.

It is seen from the Table 8 that, sugarcane cultivation was found to be profitable in all planting season such as adsali, pre-seasonal, suru and ratoon groups. It is observed from the table that, the per hectare sugarcane production in adsali was 130 tons while in ratoon it was 72 tons. Benefit: cost ratio was 1.8 in adsali, 1.7 in pre-seasonal, 1.7 in suru and 1.8 in ratoon sugarcane, respectively. This analysis indicated that sugarcane crop all season that is adsali, pre-seasonal suru and ratoon was profitable in Ahmednagar district of Maharashtra state.

It is concluded that all planting season sugarcane grower there is no significant difference in average family size, the overall level most of the grower are litrate, a general caste is dominating in sugarcane farming, the cropping pattern of sample sugarcane farms indicated that, farmers have allocated relatively more area under sugarcane crop as compared to other competitions crops. The analysis of per hectare profitability of sugarcane cultivation in all planting season 
indicated that cultivation of sugarcane were profitable at all the levels of cost, as indicated by into benefit: cost ratio of Adsali, preseasonal, suru and ratoon sugarcane is 1.80, $1.71,1.70,1.80$ respectively. In adsali sugarcane group highest per hectare main produce was 130 tonne and by produce was 12.70 tonne while lowest in ratoon sugarcane group main produce was 72 tonne and by produce was 7.20 tonne.

\section{References}

Amala R. and Rajagopal N. (2017) Economic analysis of cost and return, and profitability of sugarcane production in cuddalore district, Tamil nadu,
International Journal of Management, IT and Engineering, 7(8): 71-81.

Balaji Kendre. (2014) Socio-economic background and seasonal migration of sugarcane harvesting workers, International Journal of Humanity and Social Sciences, 1(2): 15-21.

Masuku M. B (2011) Determinants of Sugarcane Profitability: The Case of Smallholder Cane Growers in Swaziland, Asian Journal of Agricultural Sciences, 3(3): 210-214.

Zaidi and Abdul Munir (2015) Socioeconomic status of Sugarcane growers: A case study of Bijnor districts in western Uttar Pradesh, International Journal of Development Research 4(8): 1550-1554.

\section{How to cite this article:}

Bodakhe, G.M., S.S. More and Shelke, R.D. 2019. Socio-Economic Characteristic and Profitability of Sugarcane Cultivation in Ahmednagar District of Maharashtra, India. Int.J.Curr.Microbiol.App.Sci. 8(07): 552-564. doi: https://doi.org/10.20546/ijcmas.2019.807.068 